\section{Prevalência de anemia e fatores associados em mulheres adultas residentes em São Leopoldo, Rio Grande do Sul, Brasil}

\author{
Anemia prevalence and associated factors \\ among adult women in São Leopoldo, \\ Rio Grande do Sul, Brazil
}

\author{
1 Programa de Pós- \\ Graduação em Saúde \\ Coletiva, Universidade do \\ Vale do Rio dos Sinos, \\ São Leopoldo, Brasil. \\ 2 London School of Hygiene \\ and Tropical Medicine, \\ London, U.K. \\ Correspondência \\ M. T. A. Olinto \\ Programa de Pós-Graduação \\ em Saúde Coletiva, \\ Universidade do Vale do \\ Rio dos Sinos. \\ Av. Unisinos 950, \\ São Leopoldo, RS \\ 93022-000, Brasil. \\ mtolinto@unisinos.br
}

\begin{abstract}
A cross-sectional population-based study was conducted to investigate the prevalence of anemia and associated factors in adult women. $A$ representative sample was selected with 312 women aged 20 to 60 in São Leopoldo, Rio Grande do Sul State, Brazil. The bivariate analyses included the chi-squared test, prevalence ratios, and 95\% confidence intervals. Poisson regression was used to adjust effects in the multivariate analyses. Anemia prevalence was 19.2\% (95\%CI: 14.8-23.6) for all women in the sample. The highest prevalence was in black women (54\%). After controlling for confounding factors, the risk of anemia in black women was three times that of whites ( $P R=3.17$; 95\%CI: 1.85-5.41; $p<0.001)$. A protective effect against anemia ( $P R=0.44 ;$ 95\%CI: 0.19-1.07; $p=0.07$ ) was found in women over 50 as compared to younger women (20-29 years). These findings suggest the need for public health policies for anemia prevention in black women.
\end{abstract}

Anemia; Prevalence; Women; Risk Factors

\author{
Cristina Fabian 1 \\ Maria Teresa Anselmo Olinto 1 \\ Juvenal Soares Dias-da-Costa 1 \\ Fernanda Bairros 1 \\ Luis Carlos Nácul 2
}

\section{Introdução}

As anemias podem ser classificadas, segundo a etiologia, em três grupos: anemias cuja produção dos eritrócitos está alterada, anemias devido aumento da desnutrição dos eritrócitos (anemias hemolíticas) ou anemias devido às perdas sanguíneas. As anemias carenciais fazem parte do grupo de anemias, cuja produção dos eritrócitos está comprometida. A carência de ferro é muito freqüente no mundo. Estima-se que $90 \%$ das anemias sejam causadas por carência de ferro 1,2 . A anemia, embora encontrada em todos os países, é mais prevalente em países em desenvolvimento do que nos industrializados $2,3,4$. Os grupos populacionais mais afetados pela anemia são: mulheres grávidas, lactentes, crianças pré-escolares e mulheres não grávidas em idade fértil 5. Dados recentes apontam no Brasil prevalências de anemia de até $50 \%$ em crianças de 6 a 60 meses, 15 a $30 \%$ em gestantes, $20 \%$ em mulheres em idade fértil e $20 \%$ em adolescentes 4,5 .

A anemia afeta a coordenação, o desenvolvimento psicomotor e o aproveitamento escolar. A redução da concentração de hemoglobina compromete o transporte de oxigênio para todos os tecidos, reduzindo a capacidade de trabalho, desempenho físico e a resistência à fadiga em pessoas anêmicas. A queda de produtividade dos trabalhadores tem sido associada à anemia ferropriva 2,5,6,7. Em mulheres grávidas, a anemia está associada a maiores riscos de morbidade e 
mortalidade tanto materna quanto fetal, além do risco aumentado de baixo peso ao nascer 2,5,8 .

A elevada prevalência de anemia e o fato de suas conseqüências terem longa duração e em alguns casos serem irreversíveis fizeram com que essa deficiência constasse na agenda mundial de saúde. A Organização Mundial da Saúde (OMS) e Organização Pan-Americana da Saúde (OPAS) elegeram como prioridade o compromisso de combater a anemia em todo continente americano 5 .

No Brasil, o Ministério da Saúde, visando à redução da prevalência de anemia, propôs, em maio de 1999, o Compromisso Social para Redução da Anemia por Carência de Ferro no Brasil. Entre as diversas ações desencadeadas houve a determinação da obrigatoriedade da adição de ferro (30\% IDR 4,2mg/100g) e ácido fólico (70\% IDR ou $150 \mu \mathrm{g} / \mathrm{l00g}$ ) nas farinhas de milho e trigo. Essa medida teve como objetivo aumentar a disponibilidade de alimentos ricos nesses micronutrientes para todos os segmentos populacionais. Sendo que, a partir de junho de 2004, todas as farinhas de trigo e milho fabricadas - no Brasil ou importadas - deveriam estar fortificadas 5,9.

Através da obrigatoriedade da adição de ferro às farinhas, junto com a sistemática fiscalização pelos órgãos competentes, espera-se que a prevalência de anemia apresente queda nos próximos anos entre os diversos grupos populacionais do país. Portanto, há necessidade de informações sobre esta morbidade que possibilitem o monitoramento e a futura avaliação do impacto dessa política.

O presente estudo tem como objetivo oferecer dados de prevalência de anemia e identificar alguns fatores sócio-econômicos e demográficos associados a esta morbidade, em um dos grupos populacionais mais atingidos - as mulheres, para o período imediatamente anterior à implantação da política nacional de adição de ferro às farinhas.

\section{Metodologia}

Foi realizado um estudo transversal de base populacional com uma amostra representativa de 1.026 mulheres de 20 a 60 anos de idade residentes na zona urbana de São Leopoldo, Região do Vale do Rio dos Sinos, Rio Grande do Sul, Brasil, no ano de 2003. Esse estudo tinha como objetivo estudar diversos desfechos de saúde, dentre eles a prevalência de anemia.

Para a investigação específica da prevalência de anemia, foi calculada uma amostra considerando-se uma prevalência de $21 \%$ de anemia em mulheres adultas 10 , um erro de $5 \%$ e um nível de confiança de 95\%. O tamanho da amostra estimado foi de 254 mulheres, prevendo-se mais $15 \%$ para possíveis perdas/recusas, totalizando 292 mulheres.

O processo de amostragem foi realizado em diversas etapas visando atender os objetivos do estudo. Foi realizada uma amostra sistemática para a seleção de 40 setores censitários entre os 270 existentes na zona urbana do município. Na definição do número de domicílios a serem visitados considerou-se uma proporção de mulheres na faixa etária de interesse de $28,2 \%$ e um número médio de pessoas por domicílios de 3,35 11,12. Assim, foram visitados 36 domicílios por setor.

Para cada um dos setores censitários, foram sorteados aleatoriamente o quarteirão e a esquina (ponto inicial) onde deveria iniciar a coleta de dados. Foram selecionadas 1.026 mulheres de 20 a 60 anos residentes nestes domicílios. Em seguida, realizou-se uma amostragem proporcional ao tamanho do setor selecionando-se aquelas que iriam realizar os exames bioquímicos para anemia. Para atingir o número de mulheres estimado foi considerada uma média de 27 mulheres selecionadas/setor e uma amostra proporcional de $30 \%$, prevendo a necessidade média de oito exames/setor. Devido a possíveis perdas e recusas, as dez primeiras mulheres entrevistadas em cada setor que não estivessem grávidas foram convidadas a participar da coleta de sangue para a investigação de anemia. Elas recebiam um cartão de identificação e as passagens de transporte para deslocarem-se até o Laboratório Municipal de Análises Clínicas. Mesmo após três tentativas por parte dos supervisores da pesquisa, $25 \%$ delas recusaram-se se deslocar até o laboratório e, portanto, para essas mulheres a coleta de sangue foi realizada no próprio domicílio. Nesses casos, o material utilizado foi cedido pelo laboratório e coletado dentro dos procedimentos técnicos estabelecidos pela instituição. Ao final, com $1 \%$ de perdas e recusas, totalizaram-se 312 mulheres na subamostra para o estudo de anemia.

A presença de anemia foi avaliada através da determinação da concentração de hemoglobina no sangue venoso pelo método de cianometahemoglobina. Esta técnica é recomendada pelo Comitê Internacional de Padronização em Hematologia (ICSH) como padrão de referência para dosagem de hemoglobina ${ }^{13}$. A anemia foi definida a partir da concentração de hemoglobina $(\mathrm{Hb})$ $<12 \mathrm{~g} /$ dl de acordo com os critérios da OMS 2 para mulheres adultas. As informações sobre as características sócio-econômicas e demográficas da amostra foram coletadas através de questionários padronizados, pré-codificados e pré-testados, aplicados por entrevistadores submetidos a um programa de treinamento. As seguintes variáveis 
foram utilizadas para descrever a amostra: classe econômica, através da classificação da Associação Nacional de Empresas de Pesquisa (ANEP) 14; renda per capita, através da informação da renda no último mês de cada componente familiar; escolaridade, coletada em anos completos de estudo e categorizada segundo ANEP; idade, coletada em anos completos no momento da entrevista e categorizada em faixa de 10 anos; cor da pele, observada pelo entrevistador e assinalada como branca, parda/mista ou negra; e estado civil, informado pela entrevistada.

A entrada dos dados foi realizada através do programa Epi Info 6.0 (Centers for Disease Control and Prevention, Atlanta, Estados Unidos), em dupla digitação para posterior comparação - possibilitando a verificação de inconsistências. As análises foram realizadas no SPSS versão 11.0 para Windows (SPSS Inc., Chicago, Estados Unidos) e Stata 7.0 (Stata Corp., College Station, Estados Unidos). As etapas da análise incluíram respectivamente: descrição da amostra, análise bivariada das características sócio-econômicas e demográficas com anemia (teste do qui-quadrado, razões de prevalência - RP - e intervalos de confiança de 95\% - IC95\%) e análise multivariada. A análise multivariada foi realizada através de regressão de Poisson com variância robusta, utilizando como variável dependente a anemia e como variáveis independentes aquelas com nível de significância estatística do valor de $\mathrm{p} \leq 0,10$ nas análises bivariadas. Ao final da análise, as variáveis com pelo menos uma categoria com $\mathrm{p}$ valor $<0,05$ no teste de Wald foram consideradas associações estatisticamente significantes com o desfecho. O efeito do delineamento amostral para o desfecho "anemia" nas mulheres entre 20 a 60 anos de idade foi de 1,29, portanto, as etapas da análise levaram em consideração esse efeito através do comando svy do Stata.

O projeto de pesquisa foi aprovado pelo Comitê de Ética e Pesquisa da Faculdade Federal de Medicina de Pelotas. Após esclarecimentos sobre a pesquisa, as mulheres que aceitaram participar do estudo assinaram o Termo de Consentimento Livre e Esclarecido. Todas as participantes receberam por telefone ou carta os resultados dos exames bioquímicos, estando disponível uma cópia no laboratório. Para todas mulheres que apresentaram resultados da concentração de hemoglobina inferior ao desejável foi recomendada a busca de serviços de saúde.

\section{Resultados}

Foram selecionadas 315 mulheres, mas após várias tentativas de contato, $1 \%$ delas não realiza- ram a coleta de sangue, totalizando 312 mulheres. Essas mulheres tinham em média 39,5 anos de idade (desvio-padrão $=10,8$ anos), sendo que $81 \%$ estavam em idade reprodutiva. Cerca de $40 \%$ da amostra pertenciam à classe econômica C, $80 \%$ possuíam renda familiar per capita menor ou igual a três salários mínimo e mais de $80 \%$ das mulheres foram consideradas como tendo a cor da pele branca (Tabela 1 ).

A prevalência de anemia nas mulheres investigadas foi de 19,2\% (IC95\%: 14,8-23,6). Em relação à idade, observa-se que as maiores prevalências de anemia foram entre as mulheres consideradas adultas jovens ( 20 a 29 anos) e ao final da vida reprodutiva (40 a 49 anos). Restringindo-se a amostra apenas às mulheres em idade reprodutiva $(\mathrm{n}=252)$, a prevalência de anemia foi de 21,4\% (IC95\%: 16,3-26,5).

As mulheres pertencentes às classes econômicas extremas, classe $A+B$ e classes $D+E$ apresentaram maiores prevalências de anemia, respectivamente, $20,6 \%$ e $25,6 \%$ do que aquelas da classe C (Tabela 1). As médias e seus desvios-padrão de concentração de hemoglobina para as mulheres brancas, pardas/mistas e negras foram, respectivamente, $12,8 \mathrm{~g} / \mathrm{dl}(1,06), 12,8 \mathrm{~g} / \mathrm{dl}(1,97) \mathrm{e}$ $12,0 \mathrm{~g} / \mathrm{dl}(1,08)$, com $\mathrm{p}$ valor $=0,048$ para tendência linear e um desvio da linearidade não significativo estatisticamente (dados não apresentados em tabela).

A Tabela 2 apresenta as razões de prevalências, brutas e ajustadas, para as variáveis sócioeconômicas e demográficas da amostra. Na análise bruta, as variáveis idade, cor da pele, estado civil e classe social apresentaram associação com $\mathrm{p}$ valor $\leq 0,10$ com a anemia, portanto, foram incluídas no modelo multivariado. Após o ajuste no modelo multivariado, observa-se que as mulheres negras mantiveram aumento de risco para anemia em relação às mulheres brancas, ou se$\mathrm{ja}$, as negras apresentaram risco de anemia três vezes maior do que as mulheres brancas $(\mathrm{RP}=$ 3,17; IC95\%: 1,85-5,41; p valor < 0,001). O efeito protetor da idade entre 50 a 60 anos em relação às mulheres mais jovens (20 a 30 anos) manteve-se em cerca de 56\% (RP = 0,44; IC95\%: 0,19-1,07; p valor $=0,07$, embora limítrofe.

\section{Discussão}

A amostra para a investigação da prevalência de anemia é representativa da população de mulheres de 20 a 60 anos da zona urbana do Município de São Leopoldo, obtida através de um estudo transversal de base populacional. O desfecho foi avaliado através da concentração de anemia que, embora seja 
Características demográficas, sócio-econômicas e prevalência de anemia nas mulheres adultas. São Leopoldo, Rio Grande do Sul, Brasil, 2003 ( $n=312$ ).

\begin{tabular}{|c|c|c|c|}
\hline \multirow[t]{2}{*}{ Características } & \multicolumn{2}{|c|}{ Freqüência } & \multirow{2}{*}{$\begin{array}{l}\text { Prevalência } \\
\text { de anemia }\end{array}$} \\
\hline & Absoluta & Relativa & \\
\hline \multicolumn{4}{|l|}{ Idade (anos) } \\
\hline $20-29$ & 68 & 21,8 & 22,1 \\
\hline $30-39$ & 78 & 25,0 & 14,1 \\
\hline $40-49$ & 106 & 34,0 & 26,4 \\
\hline $50-60$ & 60 & 19,2 & 10,0 \\
\hline \multicolumn{4}{|l|}{ Cor da pele } \\
\hline Branca & 260 & 83,3 & 16,9 \\
\hline Parda & 39 & 12,5 & 23,1 \\
\hline Preta & 13 & 4,2 & 53,8 \\
\hline \multicolumn{4}{|l|}{ Estado civil } \\
\hline Casada/Em união & 203 & 65,1 & 17,7 \\
\hline Viúva & 11 & 3,5 & 27,3 \\
\hline Separada/Divorciada & 33 & 10,6 & 9,1 \\
\hline Solteira & 65 & 20,8 & 27,7 \\
\hline \multicolumn{4}{|l|}{ Classe econômica (ANEP) } \\
\hline$A+B$ & 102 & 32,7 & 20,6 \\
\hline C & 127 & 40,7 & 14,2 \\
\hline$D+E$ & 82 & 26,3 & 25,6 \\
\hline \multicolumn{4}{|c|}{ Renda per capita (em salários mínimos) } \\
\hline $0,00-1,00$ & 125 & 40,3 & 23,2 \\
\hline $1,01-3,00$ & 132 & 42,6 & 13,6 \\
\hline $3,01-6,00$ & 37 & 11,9 & 21,6 \\
\hline 6,01 ou + & 16 & 5,2 & 31,3 \\
\hline \multicolumn{4}{|c|}{ Escolaridade (anos - ANEP) } \\
\hline $0-4$ & 64 & 20,9 & 20,3 \\
\hline $5-7$ & 82 & 26,8 & 14,6 \\
\hline 8-10 & 49 & 16,0 & 20,4 \\
\hline $11-13$ & 65 & 21,2 & 16,9 \\
\hline $14-23$ & 46 & 15,0 & 30,4 \\
\hline
\end{tabular}

ANEP: Associação Nacional de Empresas de Pesquisa.

o parâmetro utilizado universalmente 2,15, apresenta ampla variabilidade entre o sexo, a faixa etária e grupo étnico das pessoas 2,16,17. Este problema está minimizado neste estudo, já que a amostra foi restrita a mulheres, adultas e não grávidas. Na análise estatística essa variabilidade também foi considerada utilizando-se modelos multivariados, controlando para os potenciais fatores de confusão - no caso, idade e cor da pele.

As prevalências de anemia encontradas neste estudo, tanto para mulheres de 20 a 60 anos quanto para aquelas de 20 a 49 anos, apresentaram valores muito próximos aos da literatura. Um estudo transversal de base populacional realizado em Pelotas por Olinto et al. 10, com uma amostra de mulheres de 20 a 49 anos, não grávidas, apontou uma prevalência de anemia de 21,9\% (IC95\%: 15,0-28,8), através da concentração de hemoglobina inferior a $12 \mathrm{~g} / \mathrm{dl}$. Da mesma maneira a prevalência de anemia em nosso estudo, também, ficou muito próxima da estimativa apresentada pela OPAS e OMS: $20 \%$ para mulheres não grávidas no Brasil ${ }^{4}$. Dados gerais da América Latina e Caribe indicam uma prevalência de anemia de $40 \%$ nas mulheres grávidas e $30 \%$ nas não grávidas 3 .

No presente estudo, cerca de $1 / 5$ das mulheres de 20 a 60 anos estavam anêmicas. Essa magnitude merece atenção dos gestores públicos de saúde, pois a anemia está associada à maior mortalidade entre mulheres parturientes, 
Razões de prevalências * brutas e ajustadas das variáveis sócio-econômicas e demográficas com anemia em mulheres adultas. São Leopoldo, Rio Grande do Sul, Brasil, 2003 ( $n=312$ ).

\begin{tabular}{|c|c|c|c|c|c|c|}
\hline \multirow[t]{2}{*}{ Características } & \multicolumn{6}{|c|}{ Mulheres de $20-60$ anos } \\
\hline & RP & IC95\% & p-valor & $\mathrm{RPaj}$ ** & IC95\% & $\mathrm{p}$ valor $* \star *$ \\
\hline \multicolumn{7}{|l|}{ Idade (anos) } \\
\hline $20-29$ & 1 & - & & 1,00 & - & \\
\hline $30-39$ & 1,63 & $0,29-1,35$ & 0,064 & 0,63 & $0,31-1,27$ & 0,197 \\
\hline $40-49$ & 1,18 & $0,69-2,03$ & & 1,16 & $0,67-1,99$ & 0,600 \\
\hline $50-60$ & 0,45 & $0,21-0,96$ & & 0,44 & $0,19-1,07$ & 0,070 \\
\hline \multicolumn{7}{|l|}{ Cor da pele } \\
\hline Branca & 1 & - & & 1,00 & - & \\
\hline Parda & 1,36 & $0,70-2,63$ & 0,007 & 1,24 & $0,66-2,31$ & 0,511 \\
\hline Preta & 3,17 & $1,60-6,27$ & & 3,17 & $1,85-5,41$ & 0,000 \\
\hline \multicolumn{7}{|l|}{ Estado civil } \\
\hline Casada/Em união & 1 & - & & & & \\
\hline Viúva & 1,53 & $0,56-4,15$ & 0,075 & & & \\
\hline Separada/Divorciada & 0,51 & $0,17-1,56$ & & & & \\
\hline Solteira & 1,55 & $0,89-2,71$ & & & & \\
\hline \multicolumn{7}{|l|}{ Classe social (ANEP) } \\
\hline$A+B$ & 1 & - & & & & \\
\hline C & 1,69 & $0,36-1,32$ & 0,094 & & & \\
\hline$D+E$ & 1,26 & $0,61-2,57$ & & & & \\
\hline \multicolumn{7}{|c|}{ Renda per capita (em salários mínimos) } \\
\hline $0,00-1,00$ & 1 & - & & & & \\
\hline $1,01-3,00$ & 0,58 & $0,30-1,12$ & 0,269 & & & \\
\hline $3,01-6,00$ & 0,92 & $0,36-2,34$ & & & & \\
\hline 6,01 ou + & 1,34 & $0,49-3,61$ & & & & \\
\hline \multicolumn{7}{|c|}{ Escolaridade (anos - ANEP) } \\
\hline $0-4$ & 1 & - & & & & \\
\hline $5-7$ & 0,73 & $0,34-1,57$ & 0,465 & & & \\
\hline $8-10$ & 1,00 & $0,44-2,27$ & & & & \\
\hline $11-13$ & 0,83 & $0,37-1,87$ & & & & \\
\hline $14-23$ & 1,50 & $0,71-3,18$ & & & & \\
\hline
\end{tabular}

ANEP: Associação Nacional de Empresas de Pesquisas.

* Análise de Poisson ajustada para efeito de delineamento (ED) = 1,29;

** Modelo multivariado com RPaj (razões de prevalências ajustadas) para ED e para as variáveis com p $\leq 0,10$ na análise bivariada; permaneceram no modelo: cor da pele e idade; saíram do modelo: classe social e estado civil;

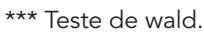

ao aumento do risco de nascimento de crianças prematuras e de baixo peso ao nascer e à mortalidade perinatal $2,5,18$. A OMS 2 propõe que a anemia deve ser considerada um problema de saúde pública quando sua prevalência exceder $5 \%$ da população. Os valores encontrados em nosso estudo são categorizados como problema de saúde pública nível médio (5 a 19,9\%) e moderado (20 a $39,9 \%$ ), se considerada a variabilidade da amostra (IC95\%).

Os resultados mostraram elevada prevalência de anemia em mulheres negras (54\%). Após ajuste no modelo multivariado de análise, o risco de anemia nas mulheres negras manteve-se três vezes maior do que nas mulheres brancas, com um limite inferior do IC95\% distante da unidade. A diferença étnica na prevalência de anemia tem sido evidenciada em alguns estudos 3,19. Entretanto, nosso resultado deve ser interpretado com cautela. O processo de amostragem foi planejando visando à representatividade, mas há a possibilidade de erro aleatório já que o tamanho da amostra foi calculado para estimar prevalência e não investigar associa- 
ções. Destaca-se, também, o baixo poder estatístico para encontrar-se diferenciais de risco de anemia segundo os fatores sócio-econômicos investigados e para detectar-se interação com cor da pele. Portanto, recomenda-se que outros estudos com amostras maiores sejam realizados.

Nosso estudo evidenciou a redução de risco de anemia nas mulheres acima de 50 anos, ou seja, após o término da vida reprodutiva. Os dados da OMS e OPAS confirmam essa tendência 2,20 .

As variáveis utilizadas neste estudo foram aquelas que usualmente são empregadas em es-

\section{Resumo}

Com o objetivo de estudar a prevalência de anemia (hemoglobina $<12 \mathrm{~g} / \mathrm{dl}$ ) e os fatores associados, foi realizado um estudo transversal de base populacional com uma amostra representativa de 312 mulheres de 20 a 60 anos residentes em São Leopoldo, Rio Grande do Sul, Brasil. As análises bivariadas incluíram testes de qui-quadrado, cálculo das razões de prevalências (RP) e intervalos de confiança de 95\% (IC 95\%). A análise multivariada foi realizada utilizando regressão de Poisson com variância robusta. Entre o total das mulheres 19,2\% (IC95\%: 14,8-23,6) estavam anêmicas. A maior prevalência ocorreu nas mulheres negras (54\%). Após controle para fatores de confusão, as mulheres negras apresentaram um risco de anemia cerca de três vezes maior comparadas com as brancas ( $R P=3,17$; IC95\%: 1,85-5,41; $p<0,001)$. Para as mulheres de 50 anos ou mais de idade foi observado um efeito protetor em comparação com as mulheres jovens $(R P=0,44$; IC95\%: 0,19-1,07; $p=0,07$ ). Os resultados do presente estudo indicam a necessidade de focalização das políticas públicas de combate à anemia, especialmente em mulheres negras.

Anemia; Prevalência; Mulheres; Fatores de Risco tudos epidemiológicos para descrever a distribuição de agravos à saúde segundo características sócio-econômicas e demográficas, ou seja, escolaridade, classe social, renda familiar, cor da pele e estado civil.

Os resultados aqui apresentados têm relevância, como fonte para futuras comparações e monitoramento da saúde na população de mulheres, por evidenciarem a necessidade da realização de ampla investigação de base populacional sobre os fatores envolvidos na determinação da anemia e por indicarem necessidade da implantação de políticas públicas direcionadas à saúde das mulheres, especialmente as negras.

\section{Colaboradores}

C. Fabian trabalhou na coleta, análise e interpretação dos dados e na redação do artigo. M. T. A. Olinto e J. S. Dias-da-Costa trabalharam em todas as etapas da pesquisa desde a elaboração do projeto até a redação final deste artigo. F. Bairros trabalhou na supervisão e coleta de dados. L. C. Nácul trabalhou na análise e interpretação dos resultados e contribuiu na redação do artigo.

\section{Agradecimentos}

Estudo financiado pela Fundação de Amparo a Pesquisa do Estado do Rio Grande do Sul (02/0645-9) e pelo Conselho Nacional de Desenvolvimento Científico e Tecnológico (Edital no. 473478/03-0) e bolsa de produtividade em pesquisa (processo no. 305906/2003-8) 


\section{Referências}

1. World Health Organization. Nutritional anemia. Geneva: World Health Organization; 1968.

2. United Nations Children's Fund/United Nations University/World Health Organization. Iron deficiency anemia: assessment, prevention, and control. Geneva: World Health Organization; 2001.

3. Center for Diseases Control and Prevention. Iron deficiency - United States, 1999-2000. MMWR Morb Mortal Wkly Rep 2002; 51:897-9.

4. Pan American Health Organization/World Health Organization. Gender, health and development in the America, 2003. http://www.paho.org/ (acessado em 15/Set/2004).

5. Ministério da Saúde. Programas de saúde: ferro. http://www.saude.gov.br (acessado em 03/ Nov/2003).

6. DeMaeyer E. Preventing and controlling iron deficiency anaemia through primary health care. Geneva: World Health Organization; 1989.

7. Li R, Chen X, Yan H, Deurenberg P, Gargy L, Hautvast J. Functional consequences of iron supplementation in iron-deficiency female cotton mill workers in Beijing, China. Am J Clin Nutr 1994; 59:90813.

8. Scholl TO, Hediger ML, Fischer RL, Shearer JW. Anemia vs iron deficiency: increased risk of preterm delivery in a prospective study. Am J Clin Nutr 1992; 32:36-9.

9. Agência Nacional de Vigilância Sanitária. Alertas técnicos. http://www.anvisa.gov.br (acessado em 03/Nov/2003).

10. Olinto MTA, Costa JSD, Gigante D, Meneses AM, Macedo S, Schwerber R, et al. Prevalência de anemia em mulheres em idade reprodutiva no sul do Brasil. Boletim da Saúde 2003; 17:135-44.

11. Instituto Brasileiro de Estatística e Geografia. Censo demográfico, 2000. http://www.ibge.gov.br (acessado em 28/Out/2003).
12. Barros FC, Victora CG. Epidemiologia da saúde infantil: um manual para diagnósticos comunitários. São Paulo: Editora Hucitec/Fundo das Nações Unidas para Infância; 1998.

13. International Committee for Standardization in Hematology. Recommendations reference method for hemoglobinometry in human blood (ICSH Standard EP 6/1: 1977) and specifications for International Haemiglobincyanide reference preparation (ICSH Standard EP 6/3:1977). J Clin Pathol 1978; 31:139-43.

14. Associação Nacional de Empresas de Pesquisa. Códigos e guias: CCEB - Critério de Classificação Econômica Brasil. http://www.anep.org.br/ (acessado em 12/Mar/2004).

15. Centers for Disease Control and Prevention. Recommendation to prevent and control iron deficiency in USA. MMWR Morb Mortal Wkly Rep 1998; 47:30.

16. Cook JD, Baynes RD, Skikne BS. Iron deficiency and the measurement of iron status. Nutr Res Rev 1992; 5:189-202.

17. Beard JL, Dawson H, Piñero DJ. Iron metabolism: a comprehensive review. Nutr Rev 1996; 54:295-317.

18. Lonnerdal B, Dewey KG. Epidemiologia da deficiência de ferro no lactente e na criança. Anais Nestlé 1996; 52:11-7.

19. Thane CW, Bates CJ, Prentice A. Risk factors for low iron intake and poor iron status in a national sample of British young peoples aged 4-18 years. Public Health Nutr 2002; 65:485-96.

20. Organização Pan-Americana da Saúde/Organização Mundial da Saúde. 130a Sessão do Comitê Executivo. Washington DC: Organização Mundial da Saúde; 2002.

Recebido em 03/Jun/2005

Versão final reapresentada em 13/Ago/2006

Aprovado em 20/Out/2006 